\title{
¿Qué es primero: desigualdades o inequidades? A propósito del caso de la mortalidad por VIH/ sida en Cali, Colombia (1986-2012)*
}

\section{What Came First, Inequalities or Inequities? Regarding the Mortality due to HIV/Aids Case in Cali, Colombia (1986-20I2)}

\section{0 que é primeiro: desigualdades ou iniquidades? A propósito do caso da mortalidade por $\mathrm{VIH} /$ sida em Cali, Colômbia (1986-2012)}

Fecha de recepción: 16-02-16 Fecha de aceptación: 04-08-16 Disponible en línea: 30-11-16 doi:10.11144/Javeriana.rgyps15-31.qpdi

Cómo citar este artículo:

Arrivillaga-Quintero M, Pérez-Flórez M, Borrero-Ramírez YE, Zea MC, Holguín-Ruiz J, Ross MW. ¿Qué es primero: desigualdades o inequidades? A propósito del caso de la mortalidad por VIH/sida en Cali, Colombia (1986-2012). Rev. Gerenc. Polit. Salud. 2016; 15(31): 216-231. http://dx.doi.org/10.11144/ Javeriana.rgyps15-31.qpdi

\author{
Marcela Arrivillaga-Quintero** \\ Mauricio Pérez-Flórez*** \\ Yadira Eugenia Borrero-Ramírez $* * * *$ \\ María Cecilia-Zea***** \\ Jorge Holguín-Ruiz $* * * * * *$ \\ Michael W. Ross $* * * * * * *$
}

\footnotetext{
Artículo de investigación derivado del proyecto Diseño e implementación de un Centro de Investigación en Desigualdades e Inequidades Sociales y en Salud (CIDSSA), con énfasis en el Pacífico colombiano. Fase 1. 15/01/2015- 17/12/2016. Financiado por la Pontificia Universidad Javeriana, Cali, Colombia.

** Psicóloga, magíster en Educación, Ph. D. en Salud Pública, directora del Departamento de Salud Pública y Epidemiología de la Pontificia Universidad Javeriana, Cali, Colombia. Dirección: Calle 18 No. 118-250 Cali, Colombia. Tel: +5723218200 Ext. 8681. Correo electrónico: marceq@javerianacali.edu.co

**** Estadístico, magíster en Epidemiología, profesor del Departamento de Salud Pública y Epidemiología de la Pontificia Universidad Javeriana, Cali, Colombia. Correo electrónico: mauricioperez@javerianacali.edu.co

**** MD, magíster en Sociología, Ph. D. en Salud Pública, docente e investigadora del Grupo Gestión y Políticas de Salud de la Facultad Nacional de Salud Pública de la Universidad de Antioquia, Medellín, Colombia. Correo electrónico: yadira.borrero@udea.edu.co

****** Psicóloga, Ph. D., profesora del Departamento de Psicología de George Washington University, Washington D. C. 20052, directora del Latino Health Research Center, codirectora del Social and Behavioral Sciences Core DC Developmental Center for AIDS Research (CFAR), Estados Unidos. Correo electrónico: zea@gwu.edu

******* MD, magíster en Epidemiología, profesor del Departamento de Salud Pública y Epidemiología de la Pontificia Universidad Javeriana, Cali, Colombia. Correo electrónico: jholguinr@javerianacali.edu.co

${ }^{* * * * * * *}$ Psicólogo, máster en Salud Pública, MHPEd, Ph. D., profesor y Joycelyn Elders Chair of Sexual Health Education del Programa en Sexualidad Humana del Departamento de Medicina Familiar de University of Minnesota, Estados Unidos. Correo electrónico: mwross@umn.edu
} 


\section{Resumen}

Este estudio tuvo como objetivo analizar la mortalidad por VIH/sida en Cali, Colombia, en el periodo 1986-2012, y a partir de ahí, comprender, desde el punto de vista conceptual, los determinantes sociales relacionados. Se llevó a cabo un estudio observacional, longitudinal, retrospectivo, con un total de 14192 casos. Se encontró una tendencia creciente de la tasa de mortalidad, en especial a partir del año 1996; en el 2011 se presentó la mayor tasa de todo el periodo. La medición de la desigualdad reveló que se han producido más muertes en estratos medios y bajos, en comparación con el estrato alto. Este estudio aporta evidencia sobre las desigualdades persistentes en la mortalidad por $\mathrm{VIH} /$ sida como expresión de inequidades de tipo estructural en la ciudad de Cali. En la perspectiva de Breilh, son procesos críticos de determinación económica y social que pudieron afectar la mortalidad, especialmente de los grupos con más vulnerados socialmente.

Palabras clave: VIH/sida; mortalidad; desigualdades en salud; inequidad social Abstract
The purpose of this study was to analyze the mortality due to HIV/Aids in Cali, Colombia, in the $1986-$
We carried, and, from there, understand from a conceptual point of view the related social determinants.
growing tendency in the mortality rate, particularly starting in 1996. The higher rate for the whole period
was seen in 2011. The inequality measure revealed a higher number of deaths in lower and middle social
strata, when compared with the high strata. This study provides evidence on the persistent inequalities in
the mortality due to HIV/Aids, as an expression of the structural inequalities present in Cali. In Breilh's
perspective, critical social and economic determination processes could have affected mortality, especially
in the groups whose social rights have been violated the most.

Keywords: HIV/Aids; mortality; health inequalities; social inequality

\section{Resumo}

Este estudo teve como objetivo analisar a mortalidade por VIH/sida em Cali, Colômbia, no período 19862012, e a partir daí, compreender desde o ponto de vista conceitual, as determinantes sociais relacionadas. Um estudo observacional, longitudinal, retrospectivo, com um total de 14192 casos foi realizado. Encontrara-se uma tendência crescente da taxa de mortalidade, em especial a partir do ano 1996; no ano 2011 apresentou-se a maior taxa do período tudo. A medição da desigualdade revelou que foram produzidas mais mortes nas camadas sociais medias e baixas, em comparação com as altas. Este estudo fornece evidencia sobre as desigualdades persistentes na mortalidade por VIH/sida, como expressão de iniquidades de tipo estrutural na cidade de Cali. Na perspectiva de Breilh, são processos críticos de determinação económica e social que puderam afetar a mortalidade, especialmente dos grupos com mais vulnerados socialmente.

Palavras-chave: VIH/sida; mortalidade; desigualdades em saúde; iniquidade social 


\section{Introducción}

Una de las discusiones más importantes de la salud pública contemporánea es el problema de las desigualdades y de las inequidades sociales y su relación con la situación de morbilidad y mortalidad de las poblaciones. La preocupación por el vínculo existente entre las condiciones de vida y la salud se empieza a instalar desde el siglo XIX, vinculada a las transformaciones de la emergente sociedad industrial, con pensadores como Virchow y Villarmé; en el siglo XX se potencia a partir de la década de los setenta con los aportes de la medicina social latinoamericana (1).

Posteriormente, con contribuciones de investigadores como Diderichsen, Evans, Whitehead, Hallqvis, Marmot, Krieger y Farmer en Europa y Estados Unidos (2-7), y los desarrollos de representantes de la salud pública y la salud colectiva en América Latina como Breilh y De Almeida Filho, entre otros (8-12). En institucional, con tendencia claramente anglosajona, y no latinoamericana, este discurso histórico se incorporó oficialmente en marzo del 2005 en la Organización Mundial de Salud (OMS), a través de la Comisión de Determinantes Sociales de la Salud, encabezada por Michael Marmot.

La investigación en el campo de las desigualdades y las inequidades sociales ha estado orientada a la identificación de diferencias sistemáticas en uno o más aspectos de la salud entre grupos o subgrupos de población, definidos social, económica, demográfica o geográficamente. De esta manera, los estudios en este campo se vinculan de manera casi natural a la epidemia del VIH/sida, dadas las desoladoras tasas de mortalidad en África subsahariana, donde la pobreza y las condiciones sanitarias han sido claros determinantes de la expansión de la infección.
En América Latina, datos del 2011 muestran que la mortalidad por esta causa presenta desigualdades entre países. Mientras en Argentina, Brasil, Costa Rica, El Salvador y Chile ha disminuido, y se ha incrementado la sobrevida con la introducción de políticas nacionales para ofrecer terapia antirretroviral gratuita, en otros como Venezuela y México las tasas de mortalidad han aumentado. No obstante, aun en los países donde han bajado las tasas, se han reportado desigualdades y mayor mortalidad cuando se analizan datos por sexo, subgrupos de edad y orientación sexual (13).

En Colombia, el campo de la investigación de las desigualdades sociales es relativamente reciente. En salud, los estudios en esta materia se hicieron visibles en publicaciones científicas desde el año 2006 en temas como vacunación $(14,15)$, obesidad (16), morbilidad en enfermedades crónicas (17), atención odontológica (18) y acceso a servicios de salud (19). Las investigaciones enfocadas en el análisis de la desigualdad en la mortalidad por causas específicas son limitadas y no tienen alcance en el contexto de los municipios, lo cual dificulta a las autoridades locales tanto la evaluación de la magnitud como la toma de decisiones en salud pública para reducir o eliminar las brechas relacionadas con resultados en salud.

En cuanto al VIH/sida, datos oficiales revelan que la tasa de mortalidad en Colombia ha descendido de 5.21 en el 2009 a 4.53 por 100000 habitantes en el 2011. Sin embargo, se encuentran desigualdades cuando se comparan los diferentes departamentos del país. Para el año 2011, aquellos con mayor tasa de mortalidad por sida fueron Quindío con 15.56, Risaralda con 8.6 y Valle del Cauca con 7.9; en todos hay mayor mortalidad en hombres que en mujeres (20). En todo caso, lo que ha mostrado la literatura científica es que las desigualdades, bien sean geográficas, 
de género o socioeconómicas, se asocian con dificultades para el acceso a los tratamientos y la adherencia terapéutica, la aparición de comorbilidades y el mayor riesgo de mortalidad (21-23). Esto se agudiza cuando se trata de los grupos más desfavorecidos en la jerarquía social y en particular en población afrodescendiente (24).

La tradición de los estudios sobre equidad en salud ha tenido como marco de referencia los planteamientos de Margaret Whitehead en 1991, quien sostuvo que la inequidad en salud se refiere a diferencias socialmente producidas, evitables, innecesarias e injustas (25). La autora propone que para calificar una situación como inequitativa es necesario examinar las causas que la han creado y juzgarlas como injustas, y que la prueba crucial para saber si las diferencias en los resultados de salud son consideradas injustas depende en gran medida de si las personas eligieron la situación que causó la mala salud o si ello estuvo fundamentalmente fuera de su control directo.

Por su parte, en la visión de la salud pública que prevalece en América Latina, en particular a partir del pensamiento crítico de Jaime Breilh en Ecuador, se ha propuesto un debate similar en términos morales y éticos. No obstante, la consideración de si la inequidad es injusta no se deriva del juicio sobre esta, ni tampoco de las elecciones personales, sino que se gesta en la estructura propia de las sociedades clasistas. De esta manera, Breilh (9-11) plantea que este tipo de estructuras sociales ha configurado a lo largo de la historia tres fuentes de inequidad que se originan en la acumulación y en la concentración del poder; estas son: la condición de género, la etnia y la clase social. Para el autor, la inequidad es la apropiación y concentración del poder en unas clases o géneros o etnias particulares, mientras que la desigualdad es la expresión observable, típica y grupal de tales inequidades; en otras palabras, una evidencia empírica que se hace verificable en las estadísticas y que revela la distribución y el acceso desigual de las personas a bienes y servicios.

A pesar de los avances en el estudio de la epidemia del VIH en América Latina, no se encuentran análisis que expliciten las relaciones entre las características de la mortalidad por esta causa y la posible presencia de desigualdades como expresión de inequidades sociales de tipo estructural presentes en los países de la región. Así, este estudio, utilizando los datos sobre mortalidad por VIH/ sida en Cali, Colombia entre 1986 y 2012, tuvo como objetivos describir la tendencia y las características en la mortalidad en el periodo observado, describir las determinaciones sociales que se le vinculan y estimar los niveles de desigualdad en la mortalidad, de acuerdo con el estrato socioeconómico, máximo indicador de referencia para el país. La perspectiva para discutir los hallazgos es la propuesta por Jaime Breilh en 1998 y en el 2003, teniendo como pregunta central la siguiente: ¿cuáles son las tendencias y los procesos críticos relacionados con las desigualdades e inequidades sociales en la mortalidad por VIH/sida en la ciudad de Cali, Colombia, durante el periodo 1986-2012?

\section{Método}

\section{Tipo de estudio}

Se realizó un estudio observacional, longitudinal, retrospectivo.

\section{Fuentes de información}

Se utilizaron los registros de casos de VIH/ sida reportados al Sistema Nacional de Vigilancia en Salud Pública (Sivigila) en el 
periodo 1986 a 2012 en Cali, Colombia. La base de datos suministrada oficialmente por la Secretaría de Salud Municipal de Cali no contenía información básica que permitiera la identificación de las personas. Luego de la depuración de datos se obtuvo un total de 14192 casos reportados en adultos mayores de 18 años.

Variables. Las variables independientes fueron sexo, escolaridad, tipo de afiliación en salud, orientación sexual, estrato socioeconómico y presencia de comorbilidades (TBC, neumocistis, criptococosis, candidiasis y toxoplasma). La variable dependiente fue mortalidad por VIH/sida. Para las comorbilidades se creó una variable llamada "índice de comorbilidades" que contaba el número de enfermedades que presentaron las personas reportadas, con valores entre 0 y 4 . Para el análisis de sobrevida se utilizó el número de días transcurridos entre el diagnóstico y el fallecimiento.

Para determinar desigualdades sociales en la mortalidad se utilizó la variable estrato socioeconómico, establecida a partir del barrio de residencia de la persona, asignando el estrato moda del barrio definido por la Administración Municipal (26). Cabe aclarar que según el Departamento Administrativo Nacional de Estadística (DANE), el estrato socioeconómico en Colombia agrupa la población en seis grupos o estratos con características sociales y económicas similares, de acuerdo con las características físicas de la vivienda y su entorno, y puede ser utilizada como una variable proxy de calidad de vida. En sí mismo, el estrato es una variable gradiente que facilita el análisis de las desigualdades en el país, pues reparte a la población en estrato 1 (bajo-bajo), 2 (bajo), 3 (medio-bajo), 4 (medio), 5 (medio-alto) y 6 (alto). Los estratos 1, 2 y 3 son beneficiarios de subsidios gubernamentales; los estratos 5 y 6 son contribuyentes; y el estrato 4 no es beneficiario ni contribuyente.

\section{Análisis de datos}

El modelo de análisis tuvo la siguiente estructura: 1) análisis de la tendencia de la tasa de mortalidad por VIH/sida de Cali para el periodo observado, 2) caracterización de los adultos reportados por $\mathrm{VIH} /$ sida, 3) análisis de determinantes de la mortalidad por VIH/ sida, 4) análisis de sobrevida de adultos fallecidos por VIH/sida, y 5) análisis de desigualdades en la mortalidad por VIH/sida.

Para todo esto se realizó un análisis descriptivo de las variables, utilizando frecuencias absolutas y porcentajes para las variables categóricas y mediana y rango intercuartil (RI) para las variables numéricas. Debido a la proporción de registros con datos perdidos, se reportó el porcentaje y el porcentaje válido (porcentaje calculado sobre los registros con datos). Para describir los determinantes de la mortalidad se calcularon Odds Ratio(OR) univariados, por medio de una regresión logística. Posteriormente, se efectuó una regresión logística multivariada utilizando el método Backward de selección de variables y la prueba de razón de versosimilitud (LR Test) para comparar los modelos. Se realizó un análisis de sobrevida utilizando como evento la mortalidad por $\mathrm{VIH} /$ sida y el tiempo hasta el fallecimiento. Se calcularon funciones de supervivencia con las variables independientes, para lo cual se empleó el método Kaplan-Meier y se determinaron diferencias por medio de la prueba Log-rank.

Luego se determinaron las variables asociadas a la sobrevida, utilizando la regresión de Cox. Se calcularon razones de peligro (HR) univariadas y multivariadas, y se utilizó la prueba LR Test en el proceso de selección de las variables. Los análisis fueron realizados en Stata 12 y se determinaron diferencias significativas cuando el valor $\mathrm{p}<0.05$. Finalmente, para determinar desigualdades en la mortalidad por estrato socioeconómico, se calcularon las tasas de mortalidad por 100000 habitantes para cada estrato. 
Se calcularon tasas por año con la tasa anual promedio. El numerador correspondió al número de muertes obtenidas de la base de datos de la Secretaría de Salud Municipal; el denominador correspondió al número de habitantes adultos por estrato, de acuerdo con las proyecciones del DANE en el censo del 2005, utilizando la población a mitad de periodo (año 2000).

Se utilizó Epidat 4.0 para explorar las desigualdades, para lo cual se emplearon índices basados en comparaciones dos a dos, como son la diferencia de tasas, la razón de tasas y el riesgo atribuible poblacional-RAP. Adicionalmente, se exploraron índices de dispersión PearcyKeppel, Pearcy-Keppel ponderado, desviación logarítmica media-DLM, varianza entre grupos-VEG y varianza relativa entre grupos (27). En todos los análisis de las desigualdades se tomó como estrato de referencia el más alto nivel socioeconómico, esto es, el estrato 6.

\section{Resultados}

Tendencia de la tasa de mortalidad por VIH/ sida de Cali para el periodo observado

Un total de 14913 casos de VIH/sida fueron reportados a la Secretaría de Salud de Cali entre 1986 y el 2012, de los cuales el $95.2 \%$ $(\mathrm{n}=14192)$ correspondió a adultos, el $2.7 \%$ $(\mathrm{n}=408)$ a menores de edad y el $2.1 \%$ $(\mathrm{n}=313)$ no pudo ser clasificado por falta de datos. En el periodo analizado sobresale una tendencia creciente de la tasa de mortalidad entre 1996 (5.5 x 100000$)$ y el 2003 (16.8 x 100000 ); posteriormente, la tendencia es a la estabilización con cifras elevadas entre el 2004 (14.6 x 100000$)$ y el 2010 (15.5 x 100000$)$. En el 2011 se presenta la mayor tasa de todo el periodo (18.9 x 100000$)$. Finalmente, la tasa decrece en el 2012 (7.5 x 100 000) (figura 1).

\section{Características de los adultos reportados por $\mathrm{VIH} /$ sida}

La edad mediana fue de 34 años (RI: 27-43). El $75.4 \%$ de los casos fueron hombres y el $24.6 \%$ mujeres. Más de la mitad, el $54.3 \%$, reportó un nivel educativo de secundaria (12 años de escolaridad), y el $35.9 \%$ nivel de primaria (5 años de escolaridad). Con respecto al estrato socioeconómico, el $44.9 \%$ se ubicó en estrato bajo, el $47.9 \%$ en estrato medio y solo el $7.2 \%$ en estrato alto. En cuanto al tipo de afiliación al sistema de salud, el $61.4 \%$ de los casos tenía afiliación al plan contributivo (para empleados y población con capacidad de pago de la prima de seguro) y el $38.6 \%$ estaba afiliado al plan subsidiado (para población en situación de pobreza), o era lo que el sistema de salud colombiano denomina población pobre no asegurada (PPNA). En cuanto a la orientación

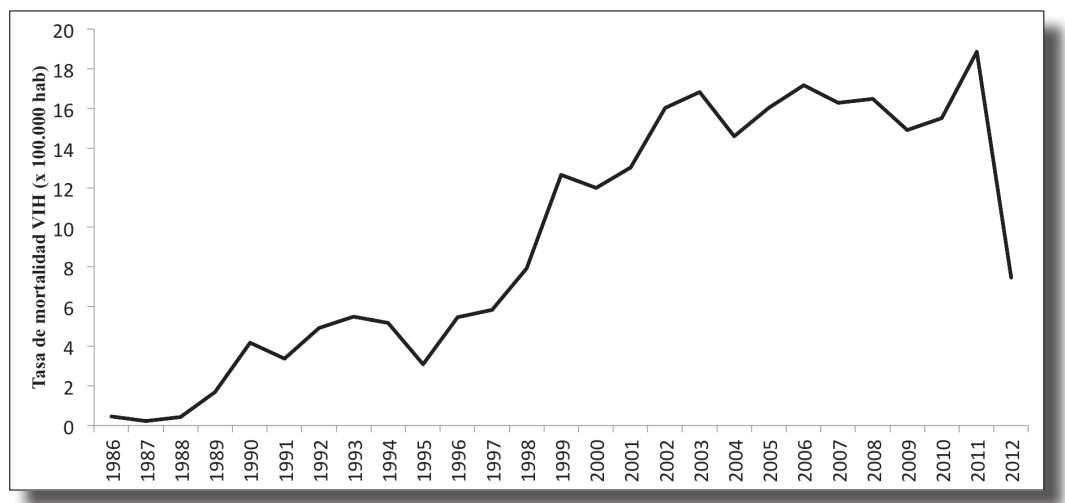

Figura 1. Tasa de mortalidad por VIH/SIDA, Cali, Colombia (1986-2012). SIVIGILA. 
Marcela Arrivillaga-Quintero • Mauricio Pérez-Flórez • Yadira Eugenia Borrero-Ramirez • María Cecilia Zea • Jorge Holguín-Ruiz • Michael W. Ross

Tabla 1. Características demográficas adultos reportados por VIH/sida Cali, Colombia. 1986-2012. SivigiLA

\begin{tabular}{|c|c|c|c|c|}
\hline \multirow{2}{*}{\multicolumn{2}{|c|}{ Variable }} & \multicolumn{3}{|c|}{ Total $n=14192$} \\
\hline & & $\mathbf{n}$ & Porcentaje & Porcentaje valido \\
\hline Edad (años) & Mediana (RI) & \multicolumn{3}{|c|}{$34(27-43)$} \\
\hline \multirow{3}{*}{ Sexo } & Masculino & 10698 & 75.4 & 75.4 \\
\hline & Femenino & 3492 & 24.6 & 24.6 \\
\hline & ND* & 2 & 0.0 & \\
\hline \multirow{5}{*}{ Escolaridad } & Ninguno/preescolar & 73 & 0.5 & 1.9 \\
\hline & Primaria & 1370 & 9.7 & 35.9 \\
\hline & Secundaria & 2073 & 14.6 & 54.3 \\
\hline & Universitaria & 304 & 2.1 & 8,0 \\
\hline & $\mathrm{ND}^{*}$ & 10372 & 73.1 & \\
\hline \multirow{4}{*}{$\begin{array}{l}\text { Estrato } \\
\text { socioeconómico }\end{array}$} & Bajo (1-2) & 3976 & 28.0 & 44.9 \\
\hline & Medio (3-4) & 4244 & 29.9 & 47.9 \\
\hline & Alto (5-6) & 638 & 4.5 & 7.2 \\
\hline & $\mathrm{ND}^{*}$ & 5334 & 37.6 & \\
\hline \multirow{3}{*}{$\begin{array}{l}\text { Tipo de } \\
\text { afiliación en } \\
\text { salud }\end{array}$} & Contributivo & 6526 & 46.0 & 61.4 \\
\hline & Subsidiado y PPNA & 4111 & 29.0 & 38.6 \\
\hline & ND* & 3555 & 25.0 & \\
\hline \multirow{4}{*}{$\begin{array}{l}\text { Orientación } \\
\text { sexual }\end{array}$} & Heterosexual & 7013 & 49.4 & 67.4 \\
\hline & Homosexual & 2580 & 18.2 & 24.8 \\
\hline & Bisexual & 812 & 5.7 & 7.8 \\
\hline & $\mathrm{ND}^{*}$ & 3787 & 26.7 & \\
\hline \multirow{5}{*}{ Comorbilidades } & TBC & 1689 & 11.9 & 11.9 \\
\hline & Neumocistis & 1047 & 7.4 & 7.4 \\
\hline & Criptococosis & 516 & 3.6 & 3.6 \\
\hline & Candidiasis & 927 & 6.5 & 6.5 \\
\hline & Toxoplasma & 941 & 6.6 & 6.6 \\
\hline \multirow{5}{*}{$\begin{array}{l}\text { Indicador de } \\
\text { comorbilidad }\end{array}$} & 0 & 10247 & 72.2 & 72.2 \\
\hline & 1 & 2954 & 20.8 & 20.8 \\
\hline & 2 & 825 & 5.8 & 5.8 \\
\hline & 3 & 148 & 1.0 & 1.0 \\
\hline & 4 & 18 & 0.1 & 0.1 \\
\hline
\end{tabular}

ND: no dato. El porcentaje válido se calcula sin tener en cuenta los ND 
sexual, el 24.8\% de los casos se reconoció como homosexual y el $7.8 \%$ como bisexual. Y sobre la prevalencia de comorbilidades, predominó la tuberculosis, con un $11.9 \%$. La tabla 1 muestra toda la información sobre las características demográficas de los adultos notificados.

\section{Determinantes de la mortalidad por VIH/sida}

El $22.1 \%$ de los casos reportados correspondió a fallecidos $(\mathrm{n}=3131)$. Al analizar los determinantes de la mortalidad, la regresión logística univariada mostró mayor mortalidad en personas afiliadas al plan subsidiado y PPNA (OR $=2.97$, IC95\%: 2.70-3.25), con menor nivel educación: ninguna/preescolar $(\mathrm{OR}=1.69$, IC95\%: 0.99-2.86) o primaria
$(\mathrm{OR}=1.97$, IC95\%: 1.51-2.58), y de estrato bajo (OR $=1.90, \mathrm{IC} 95 \%$ : 1.54-2.34). También se encontraron asociaciones entre mortalidad y presencia de una comorbilidad, en particular TBC, neumocistis, criptococosis o toxoplasma.

Por su parte, el análisis multivariado mostró que las variables ser hombre $(\mathrm{OR}=1.31$, IC95\%: 1.07-1.62), afiliado al plan subsidiado o persona pobre no asegurada $(\mathrm{OR}=2.02$, IC 95\%: 1.68 2.43), con baja escolaridad -ninguna/preescolar $(\mathrm{OR}=1.05, \mathrm{IC} 95 \%$ : 0.55-2.03) o primaria $(\mathrm{OR}=1.47, \mathrm{IC} 95 \%: 1.05-2.06)$, heterosexual (OR $=2.17$, IC95\%: 1.50-3.14) y con presencia de tres comorbilidades $(\mathrm{OR}=1.84$, IC95\%:1.053.21), tienen mayor riesgo de mortalidad por VIH/sida. La tabla 2 muestra datos completos.

Tabla 2. Determinantes de la MORTALIDAd (LETALidAd) POR VIH/SIDA DE ACUERdo CON LA REgRESIÓN logística univariada y multivariada. Cali, Colombia. 1986-2012. Sivigila

\begin{tabular}{|c|c|c|c|c|c|c|c|c|}
\hline \multirow[b]{2}{*}{ Variable } & \multicolumn{2}{|c|}{$\begin{array}{c}\text { Vivos } \\
11061(77.9 \%) \\
\end{array}$} & \multicolumn{2}{|c|}{$\begin{array}{c}\text { Fallecidos } \\
\mathbf{3 1 3 1}(\mathbf{2 2 . 1 \% )} \\
\end{array}$} & \multicolumn{2}{|c|}{ Univariado } & \multicolumn{2}{|c|}{ Multivariado } \\
\hline & $\mathbf{N}$ & $\%$ & $\mathbf{N}$ & $\%$ & $\begin{array}{c}\text { OR } \\
\text { (IC95\%) }\end{array}$ & Valor $\mathbf{p}$ & $\begin{array}{c}\text { OR } \\
\text { (IC95\%) }\end{array}$ & Valor $p$ \\
\hline \multicolumn{9}{|l|}{ Sexo } \\
\hline Femenino & 2866 & 25.9 & 626 & 20.0 & 1 & & & \\
\hline Masculino & 8193 & 74.0 & 2505 & 80.0 & $\begin{array}{c}1.40 \\
(1.27-1.54)\end{array}$ & $<0.001$ & $\begin{array}{c}1.31 \\
(1.07-1.62)\end{array}$ & 0.010 \\
\hline ND & 2 & & 0 & & & & & \\
\hline \multicolumn{9}{|l|}{ Escolaridad } \\
\hline $\begin{array}{l}\text { Ninguna/ } \\
\text { preescolar }\end{array}$ & 43 & 1.7 & 30 & 2.3 & $\begin{array}{c}1.69 \\
(0.99-2.86)\end{array}$ & 0.053 & $\begin{array}{c}1.05 \\
(0.55-2.03)\end{array}$ & 0.871 \\
\hline Primaria & 754 & 30.0 & 616 & 47.1 & $\begin{array}{c}1.97 \\
(1.51-2.58)\end{array}$ & $<0.001$ & $\begin{array}{c}1.47 \\
(1.05-2.06)\end{array}$ & 0.024 \\
\hline Secundaria & 1501 & 59.7 & 572 & 43.8 & $\begin{array}{c}0.92 \\
(0.71-1.20)\end{array}$ & 0.541 & $\begin{array}{c}0.68 \\
(0.49-0.94)\end{array}$ & 0.021 \\
\hline Universitaria & 215 & 8.6 & 89 & 6.8 & 1 & & & \\
\hline ND & 8548 & & 1824 & & & & & \\
\hline \multicolumn{9}{|c|}{ Estrato socioeconómico } \\
\hline Bajo (1-2) & 2771 & 42.4 & 1205 & 51.9 & $\begin{array}{c}1.90 \\
(1.54-2.34)\end{array}$ & $<0.001$ & & \\
\hline Medio (3-4) & 3250 & 49.7 & 994 & 42.9 & $\begin{array}{c}1.33 \\
(1.08-1.65)\end{array}$ & 0.008 & & \\
\hline Alto (5-6) & 519 & 7.9 & 119 & 5.1 & 1 & & & \\
\hline ND & 4521 & & 813 & & & & & \\
\hline
\end{tabular}


Marcela Arrivillaga-Quintero • Mauricio Pérez-Flórez • Yadira Eugenia Borrero-Ramirez • Maria Cecilia Zea • Jorge Holguín-Ruiz • Michael W. Ross

\begin{tabular}{|c|c|c|c|c|c|c|c|c|}
\hline \multirow[b]{2}{*}{ Variable } & \multicolumn{2}{|c|}{$\begin{array}{c}\text { Vivos } \\
11061(77.9 \%)\end{array}$} & \multicolumn{2}{|c|}{$\begin{array}{c}\text { Fallecidos } \\
3131(22.1 \%)\end{array}$} & \multicolumn{2}{|c|}{ Univariado } & \multicolumn{2}{|c|}{ Multivariado } \\
\hline & $\mathbf{N}$ & $\%$ & $\mathbf{N}$ & $\%$ & $\begin{array}{c}\text { OR } \\
\text { (IC95\%) }\end{array}$ & Valor $\mathbf{p}$ & $\begin{array}{c}\text { OR } \\
\text { (IC95\%) }\end{array}$ & Valor $\mathbf{p}$ \\
\hline \multicolumn{9}{|c|}{ Tipo de afiliación en salud } \\
\hline Contributivo & 5513 & 67.5 & 1013 & 41.1 & & & & \\
\hline $\begin{array}{l}\text { Subsidiado y } \\
\text { PPNA }\end{array}$ & 2661 & 32.5 & 1450 & 58.9 & $\begin{array}{c}2.97 \\
(2.70-3.25)\end{array}$ & $<0.001$ & $\begin{array}{c}2.02 \\
(1.68-2.43)\end{array}$ & $<0.001$ \\
\hline ND & 2887 & & 668 & & & & & \\
\hline \multicolumn{9}{|c|}{ Orientación sexual } \\
\hline Heterosexual & 5492 & 65.3 & 1521 & 76.2 & $\begin{array}{c}1.93 \\
(1.55-2.39)\end{array}$ & $<0.001$ & $\begin{array}{c}2.17 \\
(1.50-3.14)\end{array}$ & $<0.001$ \\
\hline Homosexual & 2207 & 26.3 & 373 & 18.7 & $\begin{array}{c}1.18 \\
(0.93-1.49)\end{array}$ & $<0.001$ & $\begin{array}{c}1.05 \\
(0.71-1.56)\end{array}$ & 0.812 \\
\hline Bisexual & 710 & 8.4 & 102 & 5.1 & 1 & & & \\
\hline ND & 2652 & & 1135 & & & & & \\
\hline \multicolumn{9}{|c|}{ Comorbilidades } \\
\hline TBC & 1082 & 9.8 & 607 & 19.4 & $\begin{array}{c}2.22 \\
(1.99-2.47)\end{array}$ & $<0.001$ & & \\
\hline Neumocistis & 625 & 5.7 & 422 & 13.5 & $\begin{array}{c}2.60 \\
(2.28-2.96)\end{array}$ & $<0.001$ & & \\
\hline Criptococosis & 330 & 3.0 & 186 & 5.9 & $\begin{array}{c}2.05 \\
(1.71-2.47)\end{array}$ & $<0.001$ & & \\
\hline Candidiasis & 668 & 6.0 & 259 & 8.3 & $\begin{array}{c}1.40 \\
(1.21-1.63)\end{array}$ & $<0.001$ & & \\
\hline Toxoplasma & 613 & 5.5 & 328 & 10.5 & $\begin{array}{c}1.99 \\
(1.73-2.30)\end{array}$ & $<0.001$ & & \\
\hline \multicolumn{9}{|c|}{ Indicador de comorbilidad } \\
\hline 0 & 8518 & 77.0 & 1729 & 55.2 & 1 & & & \\
\hline 1 & 1897 & 17.2 & 1057 & 33.8 & $\begin{array}{c}2.75 \\
(2.51-3.01)\end{array}$ & $<0.001$ & $\begin{array}{c}1.46 \\
(1.20-1.77)\end{array}$ & $<0.001$ \\
\hline 2 & 532 & 4.8 & 293 & 9.4 & $\begin{array}{c}2.71 \\
(2.33-3.16)\end{array}$ & $<0.001$ & $\begin{array}{c}1.29 \\
(0.98-1.70)\end{array}$ & 0.071 \\
\hline 3 & 99 & 0.9 & 49 & 1.6 & $\begin{array}{c}2.44 \\
(1.72-3.45)\end{array}$ & $<0.001$ & $\begin{array}{c}1.84 \\
(1.05-3.21)\end{array}$ & 0.033 \\
\hline 4 & 15 & 0.1 & 3 & 0.1 & $\begin{array}{c}0.99 \\
(0.38-3.41)\end{array}$ & 0.981 & $\begin{array}{c}0.43 \\
(0.09-2.12)\end{array}$ & 0.300 \\
\hline
\end{tabular}

Análisis de sobrevida de adultos fallecidos por VIH/sida

El análisis de la sobrevida, con variablerespuesta tiempo hasta el fallecimiento, se realizó en dos fases con los datos disponibles $224(\mathrm{n}=1369)$. En el análisis univariado se encontraron diferencias estadísticamente signi- ficativas en sobrevida por sexo, escolaridad, estrato socioeconómico, tipo de afiliación en salud y orientación sexual. La sobrevida fue menor en hombres que en mujeres $(\mathrm{HR}=1.04, \mathrm{IC} 95 \%: 0.91-1.19)$. Las personas sin escolaridad o solo con educación preescolar tienen el doble de peligro de morir, 
comparadas con personas con educación universitaria $(\mathrm{HR}=2.15$, IC95\%: 1.21-3.40).

Las personas de los estratos bajo y medio tienen mayor peligro de morir $(\mathrm{HR}=1.40$, IC95\%: $1.04-1.88$ y HR = 1.96, IC95\%: $1.46-$ 2.62, respectivamente) que las personas de estrato alto. El peligro de morir fue casi el doble en personas afiliadas al plan subsidiado o PPNA, en comparación con afiliados al plan contributivo $(\mathrm{HR}=1.92$, IC95\%: 1.71-2.16). Por orientación sexual, el riesgo fue mayor en homosexuales (HR $=2.62$, IC95\%: 2.07 3.32) y heterosexuales ( $\mathrm{HR}=1.43$, IC95\%: 1.10-1.84), en comparación con bisexuales.

El análisis multivariado, por su parte, identificó mayor peligro de morir en hombres $(\mathrm{HR}=1.23$, IC95\%: 0.99-1.53), personas sin escolaridad (HR = 1.25, IC95\%:0.65-2.41), plan subsidiado y PPNA (HR $=1.89$, IC95\%: 1.55-2.29) y homosexuales ( $\mathrm{HR}=2.25$, IC95\%: 1.57-3.23). En la tabla 3 se presentan datos completos.

\section{Desigualdades en la mortalidad por VIH/sida}

El análisis de las desigualdades en las tasas de mortalidad de VIH/sida por estrato socioe- conómico se presenta en la tabla 4, en tanto que en la figura 2 se encuentra un análisis de estas desigualdades por quinquenios. Para la razón y diferencia de tasas se tomó como referencia el estrato socioeconómico 6 , esto es, la mejor situación socioeconómica en Colombia. Puede observarse cómo las tasas de mortalidad fueron más altas en los estratos bajos (1 a 3 ) y menores en los estratos altos (4 a 6). En comparación con el estrato 6, la tasa de mortalidad VIH/sida fue 2.4 veces en el estrato $1 ; 3.1$ veces en el estrato $2 ; 3.3$ veces en el estrato $3 ; 2.1$ veces en el estrato 4 y 2.1 veces en el estrato 5. La diferencia absoluta de tasas muestra un exceso de muertes (3.4 en estrato $1 ; 5.1$ en estrato $2 ; 5.6$ en estrato $3 ; 2.8$ en estrato 4 y 2.6 en estrato 5) por cada 100000 habitantes, en comparación con el estrato 6. El riesgo atribuible poblacional $(\mathrm{RAP}=4.58)$ indica que si en Cali se alcanzaran las tasas de mortalidad de VIH/sida del más alto estrato socioeconómico (estrato 6) se evitarían en promedio 5 muertes por cada 100000 adultos, lo cual representa el $65 \%$ de las muertes que se producen en la ciudad.

$\mathrm{Al}$ aplicar diferentes índices de desigualdades, se encontró que aquellos basados en

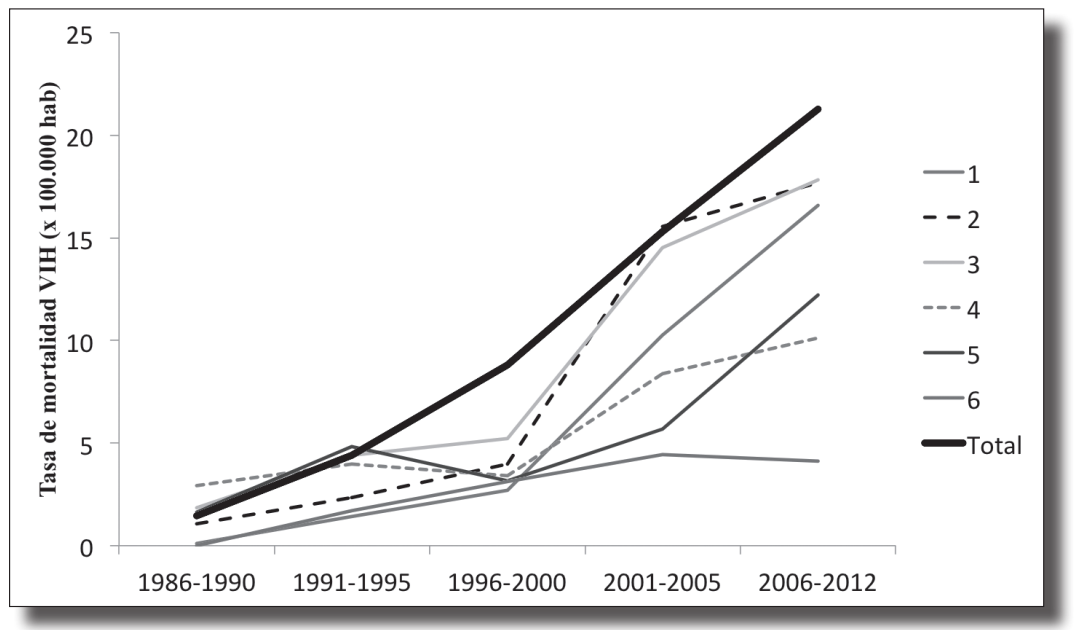

Figura 2. Tasa de mortalidad por VIH/SIDA en quinquenios por Estrato Socioeconómico. Cali, Colombia (1986-2012). SIVIGILA. 

Marcela Arrivillaga-Quintero $\bullet$ Mauricio Pérez-Flórez • Yadira Eugenia Borrero-Ramirez •
Maria Cecilia Zea $•$ Jorge Holguin-Ruiz • Michael W. Ross

Tabla 3. Análisis de sobrevida y la Regresión de Cox univariada y multivariada. Cali, Colombia. 19862012. SiVIGILA

\begin{tabular}{|c|c|c|c|c|c|c|}
\hline \multirow{2}{*}{ Variable } & \multirow{2}{*}{$n=1369$} & \multirow{2}{*}{$\begin{array}{c}\text { Log- } \\
\text { rank }\end{array}$} & \multicolumn{2}{|c|}{ Univariado } & \multicolumn{2}{|c|}{ Multivariado } \\
\hline & & & HR IC95\% & Valor $\mathbf{p}$ & HR IC95\% & Valor $\mathbf{p}$ \\
\hline Sexo & & 0.563 & & & & \\
\hline Femenino & 200 & & 1 & & 1 & \\
\hline Masculino & 1089 & & $1.04(0.91-1.19)$ & 0.537 & $1.20(1.03-1.40)$ & 0.023 \\
\hline Escolaridad & & $<0.001$ & & & & \\
\hline Ninguno/preescolar & 15 & & $2.40(1.36-4.25)$ & 0.002 & $1.77(0.99-3.17)$ & 0.054 \\
\hline Primaria & 271 & & $1.61(1.22-2.14)$ & 0.001 & $1.25(0.93-1.69)$ & 0.138 \\
\hline Secundaria & 315 & & $1.23(0.93-1.62)$ & 0.148 & $0.99(0.74-1.32)$ & 0.921 \\
\hline Universitaria & 59 & & 1 & & 1 & \\
\hline ND & 709 & & $1.57(1.20-2.05)$ & 0.001 & $1.19(0.90-1.57)$ & 0.217 \\
\hline Estrato socioeconómico & & $<0.001$ & & & & \\
\hline Bajo (1-2) & 584 & & $1.65(1.24-2.22)$ & $<0.001$ & $1.46(1.08-1.98)$ & 0.015 \\
\hline Medio (3-4) & 480 & & $1.31(0.98-1.76)$ & 0.070 & $1.16(0.86-1.56)$ & 0.346 \\
\hline Alto (5-6) & 49 & & 1 & & & \\
\hline ND & 256 & & $1.76(1.29-2.39)$ & $<0.001$ & $1.35(0.99-1.85)$ & 0.060 \\
\hline $\begin{array}{l}\text { Tipo de afiliación en } \\
\text { salud }\end{array}$ & & $<0.001$ & & & & \\
\hline Contributivo & 480 & & 1 & & 1 & \\
\hline Subsidiado y PPNA & 691 & & $1.21(1.08-1.36)$ & $<0.001$ & $1.10(0.97-1.25)$ & 0.121 \\
\hline ND & 198 & & $1.96(1.66-2.32)$ & $<0.001$ & $1.90(1.60-2.26)$ & $<0.001$ \\
\hline Orientación sexual & & $<0.001$ & & & & \\
\hline Heterosexual & 672 & & $1.55(1.22-1.97)$ & $<0.001$ & $1.60(1.25-2.04)$ & $<0.001$ \\
\hline Homosexual & 255 & & $1.17(0.90-1.51)$ & 0.234 & $1.17(0.90-1.51)$ & 0.246 \\
\hline Bisexual & 77 & & 1 & & 1 & \\
\hline ND & 365 & & $1.85(1.44-2.37)$ & $<0.001$ & $1.79(1.39-2.31)$ & $<0.001$ \\
\hline \multicolumn{7}{|l|}{ Comorbilidades } \\
\hline TBC & 321 & 0.059 & $1.13(1.00-1.28)$ & 0.059 & $1.14(1.00-1.29)$ & 0.052 \\
\hline Neumocistis & 221 & 0.533 & $1.05(0.91-1.21)$ & 0.534 & & \\
\hline Criptococosis & 110 & 0.383 & $1.09(0.90-1.33)$ & 0.385 & & \\
\hline Candidiasis & 155 & 0.250 & $1.10(0.93-1.30)$ & 0.251 & & \\
\hline Toxoplasma & 171 & 0.722 & $1.03(0.88-1.21)$ & 0.723 & & \\
\hline $\begin{array}{l}\text { Indicador de } \\
\text { comorbilidad }\end{array}$ & & 0.140 & & & & \\
\hline 0 & 662 & & 1 & & & \\
\hline 1 & 482 & & $1.12(0.99-1.26)$ & 0.067 & & \\
\hline 2 & 182 & & $1.21(1.03-1.43)$ & 0.023 & & \\
\hline 3 & 40 & & $1.12(0.82-1.54)$ & 0.479 & & \\
\hline 4 & 3 & & $0.62(0.20-1.93)$ & 0,408 & & \\
\hline
\end{tabular}

HR: Hazart ratio. ND: no dato 
¿QUÉ ES PRIMERO: DESIGUALDADES O INEQUIDADES? A PROPÓSITO DEL CASO DE LA MORTALIDAD POR VIH/SIDA EN Cali, Colombia (1986-2012)

Tabla 4. Tasas de mortalidad por VIH/Sida según estrato socioeconómico. Cali, Colombia 1986-2012

\begin{tabular}{|l|l|l|l|l|l|}
\hline $\begin{array}{l}\text { Estrato } \\
\text { socioeconómico }\end{array}$ & $\begin{array}{l}\text { Población } \\
\text { adultos* }\end{array}$ & Muertes VIH & $\begin{array}{l}\text { Tasa promedio } \\
\text { anual mortalidad } \\
\text { VIH x 100 000 }\end{array}$ & $\begin{array}{l}\text { Razón } \\
\text { de tasas }\end{array}$ & $\begin{array}{l}\text { Diferencia } \\
\text { de tasas }\end{array}$ \\
\hline 1 & 236898 & 391 & 5.9 & 2.40 & 3.44 \\
\hline 2 & 382681 & 814 & 7.6 & 3.09 & 5.14 \\
\hline 3 & 388103 & 880 & 8.1 & 3.30 & 5.64 \\
\hline 4 & 78192 & 114 & 5.2 & 2.12 & 2.75 \\
\hline 5 & 78070 & 110 & 5.0 & 2.05 & 2.58 \\
\hline 6 & 13085 & 9 & 2.5 & 1.00 & 0.00 \\
\hline
\end{tabular}

* Población adulta de mitad de periodo según proyecciones del DANE al 2000

tasas y en el concepto de dispersión, como son el índice de Pearcy-Keppel, el índice de Pearcy-Keppel ponderado, la desviación logarítmica media y la varianza entre grupos, mostraron evidentes desigualdades en la mortalidad por VIH en Cali. Esto coincide con los hallazgos respecto a las diferencias en la mortalidad por estrato socioeconómico presentadas arriba. Los índices calculados y su correspondiente definición e interpretación se describen a continuación.

- Índice de Pearcy-Keppel: establece la diferencia entre la tasa de mortalidad en cada estrato con el estrato de referencia. En este estudio se encontró este índice en 1.33 , lo que significa que hay un $33 \%$ más de mortalidad en comparación con personas del estrato 6 .

- Índice de Pearcy-Keppel ponderado: establece la diferencia en la tasa promedio de mortalidad con la del estrato de referencia, después de ajustar las tasas por el tamaño de la población en cada estrato. En este estudio se encontró el índice de 4.6, lo cual significa, considerando que la tasa promedio de mortalidad en Cali fue de 7.0 x 100000 habitantes, y que en el estrato 6 fue de $2.5 \times 100000$ habitantes, que hubo 4.6 más muertes cuando se compara con el estrato 6 .
- Desviación logarítmica media (DLM): equivalente a la varianza entre grupos en escala logarítmica (tasas en escala logarítmica). En este estudio fue de 0.02. La varianza entre grupos (VEG) fue de 1.46 y la desviación estándar (DE) 1.21. Esto significa que, en promedio, las tasas de mortalidad de los estratos difieren en 1.2 muertes x 100000 habitantes de la tasa promedio de Cali $(7.0 \times 100000$ habitantes).

\section{Discusión}

Este estudio tuvo como objetivo analizar el caso de la mortalidad por VIH/sida en Cali, Colombia, en el periodo 1986-2012, y a partir de ahí comprender, desde el punto de vista conceptual, el lugar donde se ubican los determinantes sociales relacionados con este resultado de salud. Los hallazgos se discuten a la luz del pensamiento crítico propuesto por Breilh en América Latina, respondiendo a la pregunta: ¿cuáles son las tendencias y los procesos críticos relacionados con las desigualdades e inequidades sociales en la mortalidad por VIH/sida en la ciudad de Cali, Colombia, durante el periodo 1986-2012?

Los resultados mostraron una tendencia creciente de la tasa de mortalidad por VIH/sida, en especial a partir del año 1996; en el 2011 
se presentó la mayor tasa de todo el periodo, con la cifra de 18.9 muertes por 100000 habitantes. Con respecto a los determinantes de la mortalidad, los hallazgos indicaron que hay mayor riesgo en hombres, afiliados al plan subsidiado o personas pobres no aseguradas, de bajo estrato socioeconómico, con baja escolaridad, heterosexuales y con presencia de tres comorbilidades. El análisis de sobrevida mostró una tendencia similar. Por su parte, la medición de la desigualdad, a través de todos los índices aplicados, reveló que se producen más muertes en estratos medios y bajos que en el estrato alto.

Estos hallazgos proporcionan evidencia empírica de las desigualdades en la mortalidad por VIH/sida en la ciudad de Cali, y pueden interpretarse como una expresión observable de las inequidades propias de la estructura social y económica del país y de esta ciudad. Para sustentar la hipótesis de la inequidad estructural como base de las desigualdades, planteamos los argumentos que se exponen a continuación.

Primero, Colombia, tercer país de América Latina, con una población de 46.9 millones de habitantes y con un crecimiento económico sostenido (28), mantiene amplias desigualdades sociales. Datos para el 2013 indicaron que el $30.6 \%$ de la población vive en condiciones de pobreza y el $9.1 \%$ en condiciones de pobreza extrema. El indicador aproximado de las desigualdades en la distribución del ingreso o coeficiente de Gini para este mismo año fue de 0.539 a escala nacional y 0.505 para Cali. Así, el país se ubica entre los primeros seis del mundo con mayor desigualdad (29), tendencia que se ha mantenido en la historia reciente, y que es incluso superior al promedio de América Latina. Esto indica que la sociedad colombiana tiene una inequidad estructural con respecto a la distribución de la riqueza que se concentra en los sectores más aventajados en términos socioeconómicos y que afecta la distribución de bienes y servicios en el resto de la población, en especial en los sectores más vulnerables.

Estas condiciones de inequidad han sido exploradas en estudios previos conducidos en el campo del VIH en Colombia, los cuales han reportado asociaciones con determinantes sociales de tipo estructural. De manera coincidente con los hallazgos aquí descritos, la evidencia hallada señala que la posición social, la configuración del actual sistema de salud - con planes de beneficio diferenciales para pobres y ricos-, las condiciones de infraestructura y atención sanitaria, la educación, el tipo de vivienda, la zona de residencia — urbana/rural—y la exclusión y marginación socioeconómica se vinculan significativamente con riesgos asociados a la morbilidad y a los riesgos de mortalidad $(21,30,31)$.

En segundo lugar, al revisar la tendencia en la mortalidad por VIH/sida, los hallazgos permiten elaborar explicaciones a partir del análisis del contexto socioeconómico de la ciudad de Cali durante el periodo observado. Llama especialmente la atención el incremento de la mortalidad a partir de 1996, año en que la ciudad comenzó a experimentar una fuerte recesión económica, con crecimiento negativo, que se extendió hasta el año 2005 (32). A su vez, las desigualdades en la tasa de mortalidad encontradas en este estudio, que fueron evidentemente mayores a partir del año 2000, también podrían explicarse con la crisis económica, la cual afectó más gravemente a los grupos de población más vulnerables, dado el deterioro de la calidad de vida y el difícil acceso al sistema de salud, que en Colombia depende, principalmente, de ser empleado (33).

En tercera instancia, explicaciones de los hallazgos de este estudio que sustentan la hipótesis de la inequidad estructural también 
se encuentran en el proceso de implementación del SGSSS en Colombia, con la reforma hecha por la Ley 100, aprobada en 1993 pero puesta en marcha en 1996. Uno de los primeros efectos de esta ley fue una crisis de la red pública hospitalaria entre 1998 y el 2002, caracterizada por la transformación de los anteriores hospitales públicos en Empresas Sociales del Estado (ESE), con la pretensión de lograr autosostenibilidad financiera. El resultado fue una crisis que se caracterizó por insuficiencia de recursos económicos y procesos de reorganización institucional, entre otros (34). Esta crisis hospitalaria, que afectó la prestación de los servicios de salud en todo el país, también pudo incrementar las barreras de acceso a la atención y a los medicamentos antirretrovirales, en especial de las personas con VIH/sida afiliadas al plan subsidiado o PPNA, cuya atención dependía mayoritariamente de la red pública hospitalaria.

En conjunto, los hallazgos aportan evidencia sobre las desigualdades persistentes en la mortalidad por VIH/sida como expresión de inequidades de tipo estructural. La desigual distribución de la riqueza a escala nacional y en Cali, la crítica situación económica local durante el periodo analizado, y la crisis de la red pública hospitalaria a partir de la implementación de la Ley 100 de 1993, se configuran como procesos de determinación económica y social de tipo general que pudieron afectar la mortalidad, especialmente de los grupos con mayores desventajas sociales afectados por la infección.

Ahora bien, a escala global también se encuentran investigaciones que dan cuenta de datos que apoyan la hipótesis de la inequidad estructural propuesta en este análisis. En el 2006 un estudio comparativo evidenció que la mortalidad era mayor en países de bajos ingresos que en países de altos ingresos (35). En Barcelona, España, en el año 2007 se hallaron desigualdades en la mortalidad tanto a escala individual como a escala comunitaria, en especial en contextos deprivados económicamente (36). En el 2008 en Canadá se encontró que el bajo estatus socioeconómico, también asociado al contexto, estuvo vinculado con un aumento en la mortalidad, incluso de personas que recibían terapia antirretroviral (37).

Con respecto a las limitaciones de este estudio, los autores reconocemos que pueden presentarse problemas de validez debido a los registros sin información encontrados en la base de datos del Sivigila. Sin embargo, y dado que las capitales colombianas suelen tener mejor cobertura y calidad de los datos estadísticos, los autores asumimos este análisis y sus resultados, a pesar de los vacíos de información. De lo contrario, no sería viable en el país aproximarse e investigar las desigualdades e inequidades sociales en la mortalidad en general, y en este caso a la mortalidad por $\mathrm{VIH} /$ sida. A pesar de esta limitación, impuesta por el contexto de los datos en Colombia, los resultados muestran evidencia empírica inicial que permite entender, desde un marco analítico sociocrítico, la relación entre las desigualdades en la mortalidad y las inequidades estructurales de tipo socioeconómico para la ciudad de Cali.

En cuanto a recomendaciones, proponemos atender lo prioritario, lo más urgente. Primero, establecer registros de vigilancia de salud más completos y de mejor calidad. Segundo, incluir en ellos variables claves como la pertenencia étnica y una mejor descripción de la ocupación. Tercero, desarrollar políticas y establecer mecanismos que garanticen equidad, tanto en las condiciones de vida de las poblaciones como en el interior del sistema de salud, para disminuir efectivamente las desigualdades sociales con respecto a la mortalidad por VIH y otros resultados sanitarios. Es importante implementar acciones políticas para afrontar los periodos de crisis económica y proteger así a los grupos de per- 
sonas más vulnerables que viven con VIH y evitar un mayor impacto sobre la mortalidad.

\section{Conclusión}

Por último, este artículo busca contribuir al debate sobre dos posiciones con respecto a las desigualdades versus las inequidades en salud. Si bien el enfoque de análisis presentado se basa en los desarrollos en América Latina, de la mano de Jaime Breilh, valdría la pena que futuros estudios en la región y a escala global intentaran análisis similares con los datos correspondientes. Sustentar empíricamente el asunto de la inequidad estructural como base de las desigualdades en salud, es una tarea compleja y desafiante, no solo en términos conceptuales, sino también metodológicos.

\section{Reconocimientos}

Los autores agradecen el apoyo financiero de la Pontificia Universidad Javeriana, Cali, Colombia para llevar a cabo este estudio. Además, reconocen la colaboración de la Secretaría de Salud Municipal de Cali que autorizó el uso de la base de datos del Sistema Nacional de Vigilancia en Salud Pública (Sivigila).

\section{Referencias bibliográficas}

1. Breilh J. Epidemiología: economía, medicina y política. Quito: Universidad Central; 1979.

2. Diderichsen F, Hallqvist J. Social inequalities in health: some methodological considerations for the study of social position and social context. En Arve-Pares B, editor. Inequality in health. A Swedish perspective. Estocolmo: Swedish Council for Social Research; 1998.

3. Diderichsen F, Evans T, Whitehead M. The social basis of disparities in health. En Evans T, Whitehead M, Diderichsen F, Bhuiya A, Wirt M, editores. Challenging inequities in health: From ethics to action. Nueva York: Oxford University Press; 2001.

4. Farmer P. Pathologies of power. Berkeley: University of California Press; 2003.
5. Krieger N. Epidemiology and the web of causation: has anyone seen the spider? Social Science \& Medicine. 1994; 39(7):887-903.

6. Krieger N. Proximal, distal, and the politics of causation: What's level got to do with it? American Journal of Public Health. 2008; 98(2):221-30. doi:10.2105/AJPH.2007.111278

7. Marmot M. The status sindrome: How social standing affects our health and longevity. Nueva York: Time Books; 2004.

8. Breilh J. La triple carga: Trabajo, práctica doméstica y procreación. Quito: CEAS; 1991.

9. Breilh J. La inequidad y la perspectiva de los sin poder: construcción de lo social y del género. En: Breilh J. Cuerpos, diferencias y desigualdades. Bogotá: Utópica Ediciones; 1998.

10. Breilh J. Epidemiología crítica: ciencia emancipadora e interculturalidad. Buenos Aires: Lugar Editorial; 2003.

11. Breilh J. Latin American critical ('social') epidemiology: new settings for an old dream. Int J Epidemiol. 2008; 37(4):745-50. doi: 10.1093/ije/dyn135

12. De Almeida Filho N. La ciencia tímida: Ensayos de deconstrucción de la epidemiología. Buenos Aires: Lugar Editorial; 2000.

13. Alonso González M, Martin L, Muñoz S, Jacobson JO. Patterns, trends and sex differences in HIV/ AIDS reported mortality in Latin American countries: 1996-2007. BMC Public Health. 2011; 11:605. doi:10.1186/1471-2458-11-605

14. Acosta N, Rodríguez J. Inequidad en las coberturas de vacunación infantil en Colombia, Años 2000 y 2003. Revista Salud Pública. 2006; 8(1):102-15.

15. Acosta N, Rodríguez J. Factores asociados a la inequidad en la vacunación infantil en Colombia : Revista Gerencia y Políticas de Salud. 2010; 9(18):116-23.

16. Álvarez LS, Goez JD, Carreño C. Factores sociales y económicos asociados a la obesidad : los efectos de la inequidad y la pobreza. Revista Gerencia y Políticas de Salud. 2012; 11(23):98-110.

17. Cerezo MP, Cifuentes OL, Nieto E, Parra JH. Desigualdades de la morbilidad por enfermedades crónicas según determinantes estructurales e intermediarios. Revista Gerencia y Políticas de Salud. 2012; 11(23): 165-188.

18. Rengifo H, Corchuelo J. Inequidades en la atención odontológica en una red de salud en Cali, Colombia. Revista de Salud Pública. 2009; 11(4): 526-37.

19. Guarnizo C, Agudelo C. Equidad de género en el acceso a los servicios de salud en Colombia. Revista de Salud Pública. 2008; 10(1): 44-57.

20. Colombia, Ministerio de Salud y Protección Social. Resumen de situación de la epidemia por VIH/ SIDA en Colombia, 1983 a 2011. Bogotá: UNGASS, Ministerio de Salud y Protección Social; 2012.

21. Arrivillaga M, Ross M, Useche B, Alzate ML, Correa D. Social position, gender role, and treatment adherence among Colombian women living with HIV/AIDS: social determinants of health approach. Pan American Journal of Public Health. 2009; 26(6):502-10. 
22. Fox AM. The HIV-poverty thesis re-examined: poverty, wealth or inequality as a social determinant of HIV infection in sub-Saharan Africa? Journal of Biosocial Science. 2012;44(4):459-80. doi:org/10.1017/S0021932011000745

23. Simard EP, Fransua M, Naishadham D, Jemal A. The influence of sex, race/ethnicity, and educational attainment on human immunodeficiency virus death rates among adults, 1993-2007. Archives of Internal Medicine. 2012;172(20):1591-8. doi:org/10.1001/archinternmed.2012.4508

24. Singh GK, Azuine RE, Siahpush M. Widening socioeconomic, racial, and geographic disparities in HIV/AIDS mortality in the United States, 1987-2011. Advances in Preventive Medicine. 2013; 657961. doi:10.1155/2013/657961

25. Whitehead M. The concepts and principles of equity and health. Health Promotion International. 1991; 6(3):217-28. doi:10.1093/heapro/6.3.217

26. Cali en Cifras, 2012. Departamento Administrativo de Planeación, Alcaldía de Santiago de Cali. Disponible en: http://www.cali.gov.co/planeacion/ publicaciones

27. Schneider MC, Castillo-Salgado C, Bacallao J, Loyola E, Mujica OJ, Vidaurre M, Roca Anne. Métodos de medición de las desigualdades de salud. Revista Panamericana de Salud Pública. 2002; 12(6):398-414.

28. Departamento Administrativo Nacional de Estadística (DANE). Resultados pobreza monetaria y desigualdad 2013 por departamentos. Disponible en: https:/www.dane.gov.co/files/investigaciones/ condiciones_vida/pobreza/bol_pobreza_13.pdf. 2013

29. World Bank, DANE. Boletín pobreza monetaria y multidimensional en Colombia 2011. Disponible en: http://www.dane.gov.co/files/investigaciones/ condiciones_vida/pobreza/boletin_pobreza_2011. pdf. 2011

30. Alvis N, Correa J, Carcamo A. La mortalidad por sida y su impacto económico en Cartagena de Indias, Colombia, 1995-2000. Biomédica. 2002;303316.

31. Tovar LM, Arrivillaga M. VIH/SIDA y determinantes sociales estructurales en municipios del Valle. Revista Gerencia y Políticas de Salud. 2010; 10(21):112-23.

32. USAID, FUPAD, Centro Nacional de Consultoría. Diagnóstico Cali, 2011. Bogotá: USAID, FUPAD, Centro Nacional de Consultoría; 2011.

33. Echeverry E. Indignación justa: estudios sobre la acción de tutela en salud en Medellín. Medellín: Editorial Universidad de Antioquia; 2013.

34. Borrero-Ramírez YE. Luchas por la salud en Colombia. Cali: Sello Editorial Javeriano, Universidad de Antioquia; 2014.

35. Braitstein P, Brinkhof MW, Dabis F, Schechter M, Boulle A, Miotti P, et al. Mortality of HIV1-infected patients in the first year of antiretroviral therapy: comparison between low-income and highincome countries. Lancet. 2006; 367(9513):817-24.

36. Marí-Dell'Olmo M, Rodríguez-Sanz M, GarciaOlalla P, Pasarín MI, Brugal MT, Caylà J, Borrell C. Individual and community-level effects in the socioeconomic inequalities of AIDS-related mortality in an urban area of southern Europe. Journal of Epidemiology and Community Health. 2007; 61(3): 232-40. doi:10.1136/jech.2006.048017

37. Joy R, Druyts EF, Brandson EK, Lima VD, Rustad CA, Zhang W, Hogg RS. Impact of neighborhoodlevel socioeconomic status on HIV disease progression in a universal health care setting. Journal of Acquired Immune Deficiency Syndromes. 2008; 47(4):500-5. doi:10.1097/QAI.0b013e3181648dfd 